\title{
Precisiones sobre la corrosión del acero en contacto con hormigón
}

[En relación con el trabajo "CORROSION DE TUBERIAS EN INSTALACIONES DE EDIFICACION" del Prof. Dr. J. Calleja y el Lcdo. E. Molina. Materiales de Construcción número 185 (1982) 15]

C. ANDRADE - IETCC - HIDRII

\section{$R E S U M E N$}

En los presentes comentarios se insiste en que, para evaluar la velocidad de corrosión de los metales, en la actualidad se pueden utilizar técnicas electroquimicas no destructivas que permiten seguir la evolución del proceso diariamente, o bien, en el caso de no contar con los aparatos apropiados, se puede acudir a una determinación gravimétrica de la pérdida de metal. La simple observación visual es una valiosa indicación complementaria de las técnicas antes mencionadas, pero no debe utilizarse como técnica de medida única por la subjetividad que conlleva.

También se precisan algunos aspectos sobre la corrosión que el acero desnudo y el galvanizado pueden sufrir por estar en contacto con materiales de construcción, como son la influencia de la alcalinidad $y$ de la humedad del medio $y$, fundamentalmente, se analiza la influencia oue puede ejercer el tipo de cemento en el riesgo de corrosion de ambas clases de metales.

\section{$S U M M A R Y$}

\begin{abstract}
In this paper, we emphasize that now it is possible to use some electrochemical non-destructive techniques for measuring the corrosion rate of the metals. Also, it is possible to use the gravimetric determinations if the appropriate instrumentation is not available. The simpple visual observation is a good complementary technique but it must not be used alone because of its subjectivity.
\end{abstract}

Also, we stress some aspects, such as the influence of the alkalinity and the humidity, about the corrosion, that the bare and galvanized steel, can suffer in contact with construction materials.

We analyze mainly, the influence of the type of cement on the risk of corrosion of both kinds of metals.

\section{INTRODUCCION}

En algunas regiones de nuestro país se han producido numerosos casos de ataque de tuberías de conducción de agua cuando estas tuberías están en contacto con materiales de construcción. Las causas han sido múltiples y, en general, ligadas a la presencia de cloruros en los materiales o a una ejecución defectuosa de la instalación, tanto en cuanto a su diseño, como a la puesta en obra de los distintos materiales empleados. Este problema ha obligado a la reposición completa de instalaciones de conducción de agua en bastantes edificios.

En determinadas regiones, donde los casos han sido especialmente numerosos en los últimos años, se ha sospechado, en ocasiones, que esta situación pudiera estar relacionada con la presencia de escorias siderúrgicas o cenizas volantes en los cementos PA (1) ampliamente utilizados en la edificación, en la actualidad.

Para poner de manifiesto si es o no el cemento el causante de esta mayor incidencia de accidentes sería necesario abordar, por un lado, un análisis de los deterioros ocurridos, lo 
que no es posible sin la colaboración de autoridades, colegios profesionales, constructores y usuarios, y por otro, es necesario efectuar un estudio sistemático del sistema tuberíamortero con los cementos y demás materiales utilizados en el área.

Los resultados provisionales (obtenidos al año) de un estudio sobre la influencia de cementos conteniendo escorias siderúrgicas sobre la corrosión de tuberías de acero desnudo y galvanizado han sido presentados por el Prof. Dr. J. Calleja y el Lcdo. E. Molina en el n. ${ }^{\circ} 185$ de esta revista "Materiales de Construcción" (1).

Esta interesante iniciativa que implica un volumen importante de trabajo y un encomiable deseo de clarificar situaciones ambiguas, pierde gran parte de sus posibilidades por la anticuada técnica de medida utilizada (simple observación visual) y por recurrir a una calidad de hormigón de recubrimiento, demasiado buena para reflejar la realidad de los morteros utilizados habitualmente.

Hace dos décadas eran muy pocos los investigadores que tenían aparatos y conocimientos para evaluar cuantitativamente la corrosión. Entonces no quedaba más remedio que recurrir a la observación visual, que cuando era precisa, además de valorar el "corroído" o "no corroído", medía el \% de área corroída, el número de picaduras si el ataque era localizado, o la profundidad de penetración, si era homogéneo. Investigadores más rigurosos acudían a la determinación gravimétrica de la pérdida de metal.

Desde hace 10 años el desarrollo de los potenciostatos (fuentes de tensión constante) ha llevado a poner a punto técnicas electroquímicas de medida de la corrosión metálica mucho más precisas, y que permiten hacer un seguimiento continuo y cuantitativo de la velocidad de corrosión (2) (3).

En la actualidad, la simple observación visual, sin ir acompañada de otras indicaciones más exactas, se valora con muchas precauciones, por la gran carga de subjetividad que encierra.

Además, hay materiales, como el acero desnudo, que la corrosión o no-corrosión por simple observación visual, es relativamente fácil de delimitar, dado el aparatoso volumen y color de los productos de colrosión. Pero en otros materiales, como es el caso del acero galvanizado, es justo lo contrario, ya que sus productos de corrosión son poco voluminosos, de color blanco o grisáceo y, en ocasiones, visibles sólo con observaciones al microscopio.

A continuación se comentarán algunas precisiones [expuestas ya en trabajos anteriores publicados en esta misma revista (4) (5) (6)] sobre la influencia que puede tener el tipo de cemento en la corrosión de los dos tipos de metales, el acero desnudo y el galvanizado, por el momento los más comúnmente utilizados en las conducciones de agua para la edificación.

Es frecuente al referirse a la corrosión de este tipo de tuberías, que se hable indistintamente de ambos tipos de metales, sobreentendiendo que lo que es bueno para el acero desnudo lo es también el galvanizado, lo que como se expondrá más adelante, no resulta cierto en la mayoría de los casos. Cuando se intenta delimitar los factores idóneos para la correcta estabilidad y durabilidad de las tuberías es fundamental, por lo tanto, que se especifique con claridad qué metal se considera.

\section{CORROSION DEL ACERO GALVANIZADO EN CONTACTO CON HORMIGON}

En trabajos anteriores publicados en esta misma revista (6) se ha expresado la actual 
controversia existente a nivel mundial sobre la estabilidad de armaduras de acero galvanizado en contacto con hormigón. Unos países las prohiben tajantemente como Alemania, y otros como EE.UU. obligan a su uso en determinados ambientes agresivos, como los marinos (7). Francia empieza a emplearlos incluso en hormigones pretensados (8).

Esta controversia, en cambio, no se ha planteado con las tuberías galvanizadas, tal vez porque es práctica común en otros países protegerlas del medio ambiente mediante materiales aislantes diferentes del mortero u hormigón usado habitualmente en nuestro pais.

Desde 1975 el Centro Nacional de Investigaciones Metalúrgicas (CENIM) y el Instituto Eduardo Torroja crearon un grupo de trabajo para estudiar la estabilidad del acero galvanizado en contacto con hormigón que en la actualidad configura el Equipo "Corrosión y protección de armaduras", habiendo realizado ya varias publicaciones (9) (10) (11).

Para el estudio se están utilizando técnicas de medida electroquímicas, determinaciones gravimétricas y observaciones al microscopio óptico y electrónico.

Tal y como se deduce del diagrama de Pourbaix para el $\mathrm{Zn}$ (figura 1), en presencia y en ausencia de $\mathrm{CO}_{2}$, el zinc en contacto con un electrólito de $\mathrm{pH} \simeq 12$ se corroe fuertemente y en el caso del hormigón, además, el potencial se sitúa por debajo de la línea de desprendimiento de $\mathrm{H}_{2}$ (huella más rugosa que observan J. Calleja y E. Molina (1) y que es el resultado de las burbujas de gas que quedan atrapadas por el fraguado del cemento).

Esta corrosión se visualiza también en el conocido diagrama de Roetheli, Cox y Littreal de la figura 2 , donde se aprecia que a partir de $\mathrm{pH} \simeq 12$, la corrosión del $\mathrm{Zn}$ aumenta con el $\mathrm{pH}$ de forma exponencial.

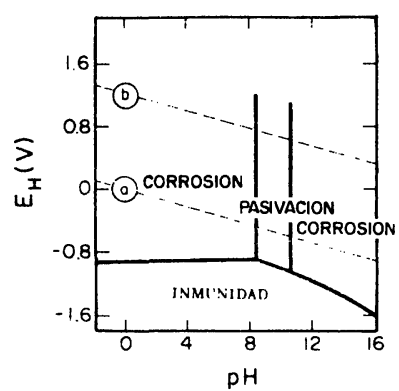

(a)

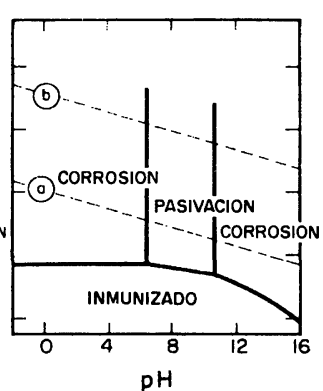

(b)

Fig. 1.-Condiciones teóricas de corrosión, inmunidad y pasivación del $\mathrm{Zn}$ a $25^{\circ} \mathrm{C}$. a) para disoluciones libres de $\mathrm{CO}_{2}$ y b) para disoluciones conteniendo $\mathrm{CO}_{2}(1 \mathrm{~mol} / l)$.

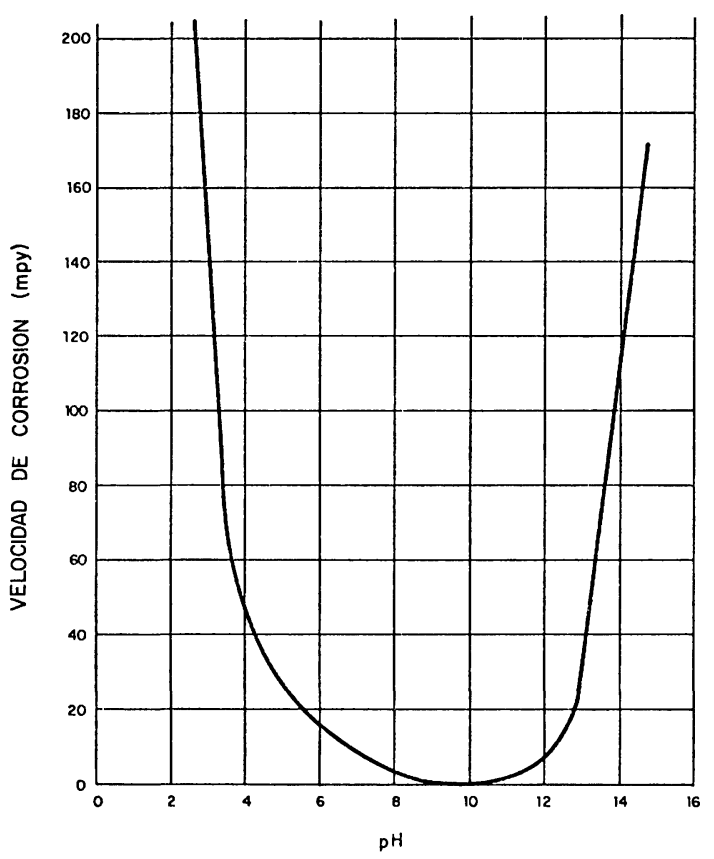

Fig. 2.-Velocidad de corrosion del $\mathrm{Zn}$ en función del $\mathrm{pH}$ del electrólito.

Según estas primeras indicaciones, se podría pensar que el $\mathrm{Zn}$ no es estable en un medio tan alcalino como el hormigón. Sin embargo, la realidad es que el producto de esta fuerte corrosión es una sustancia, el hidroxicincato cálcico, que recubre toda la superficie y que tiene propiedades pasivantes. Es precisamente en este punto donde surge la controversia, 
porque unos autores indican que el galvanizado en contacto con hormigón se pasiva, y otros aseguran que la corrosión continúa hasta la disolución total del recubrimiento.

Los resultados principales aportados por los investigadores del CENIM y del Instituto Torroja (6) (10) (11) (12), han consistido hasta el momento en la identificación y cuantificación de las tres variables responsables de la pasivación, o no, del acero galvanizado. Estas tres variables son: a) las características metalográficas del recubrimiento galvanizado, b) la humedad de conservación del hormigón y c) el tipo de cemento (concretamente su contenido en álcalis solubles).

a) El galvanizado en caliente está constituido por unas aleaciones intermetálicas Fe-Zn (figura 3), que aumentan su contenido en $\mathrm{Zn}$ desde el acero base hasta el exterior que, en general, está constituido por una capa de $\mathrm{Zn}$ puro (llamada capa $\eta$ ).

Se ha comprobado que en ausencia de $\mathrm{Cl}^{-}$es esta capa $\eta$ la que se corroe preferencialmente como muestran las figuras 4 y 5 .

Cuanto más gruesa y homogénea sea esta capa, más resistente es el galvanizado en caliente a la corrosión por electrólitos muy alcalinos.

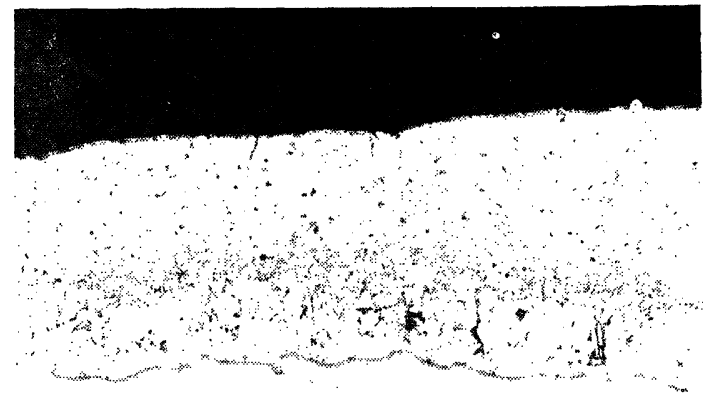

Fig. 3.-Micrografia $1 \times 300)$ de un recubrimiento galvanizado a $450^{\circ} \mathrm{C}$ que presenta una gruesa capa $\eta$ exterrna de Zn puro.

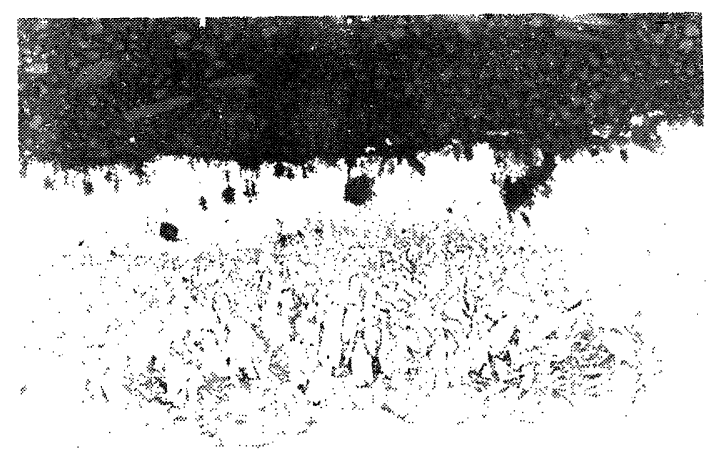

Fig. 4,.... Micrografia $1 \times 300$, de un recuisrimionto galvanizado atacado por un mortero fabricado con un cemento de elevado contenido en alcalis.

b) Se ha comprobado también que la humedad de conservación del hormigón es determinante en ocasiones, de la pasividad o actividad del galvanizado. Si el hormigón está seco la corrosión siempre es muy pequeña.

Pero si el hormigón se conserva en ambientes con $\mathrm{HR} \geqslant 60-70 \%$ (figura 6) la corrosión unas veces es alta, y otras, después de un primer momento de elevada actividad, se mantiene en valores muy pequeños (prácticamente de pasividad). Hasta el momento, no hemos podido encontrar una explicación satisfactoria a la influencia de esta variable, ni podemos predecir el sentido concreto de su actuación. Las líneas de investigación por las que estamos profundizando están relacionadas con la posibilidad de que el contenido en humedad influya en el $\mathrm{pH}$ de la fase acuosa, y con la probabilidad de que humedades relativas entre el $70 \%$ y el $100 \%$ no saturen totalmente de agua los poros del hormigón, dando lugar a fenómenos de aireación diferencial.

Lo que está claramente demostrado (figuras $6 y 7$ ) es que humedades relativas $\geqslant 60$ - 
$70 \%$ aceleran siempre la velocidad de corrosión del galvanizado, haciendola, en ocasiones, superar los límites considerados como peligrosos para la durabilidad del recubrimiento.

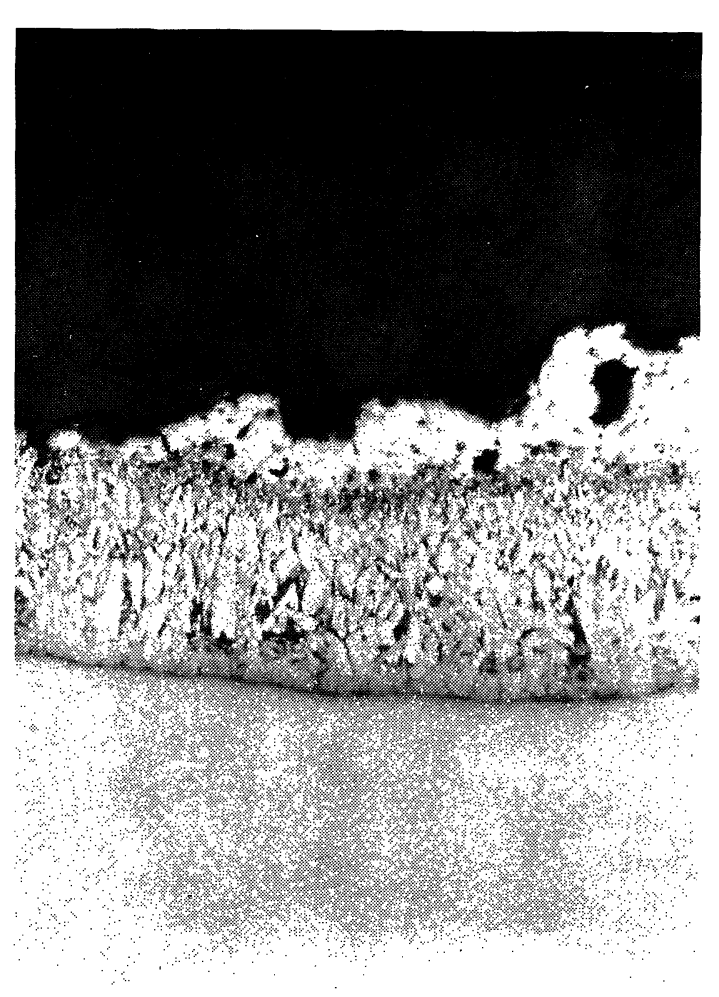

Fig. 5.--Idem. que la anterior.
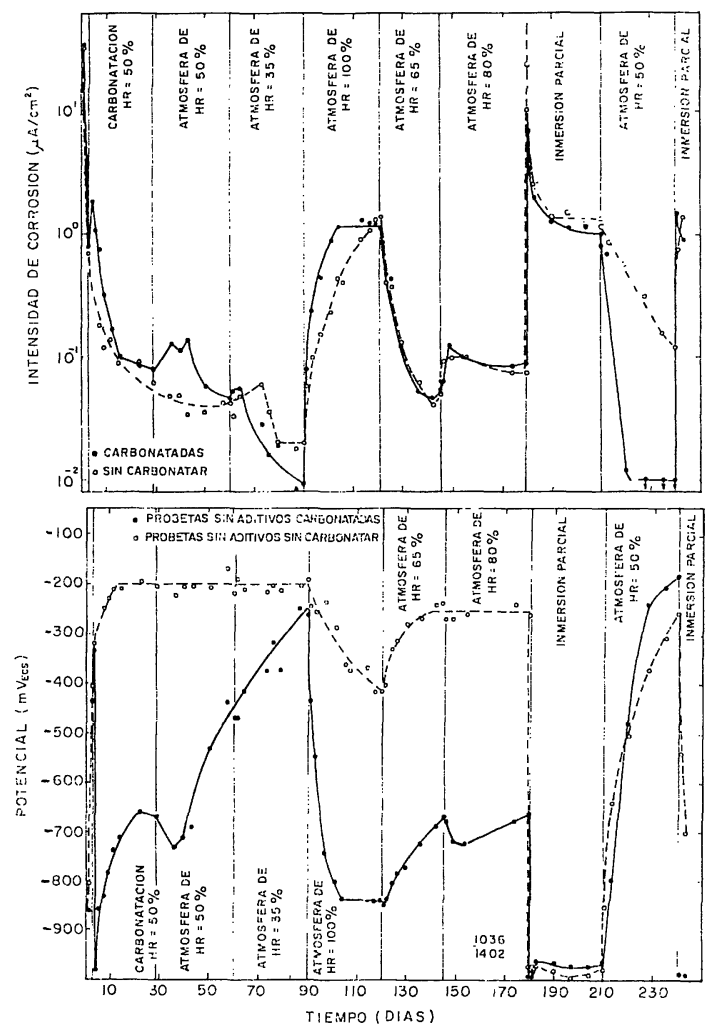

Fig. 6.-Variacion de las intensidades y los potenciales de corrosión con el tiempo de aceros para pretensado galvanizados embebidos en mortero, sin aditivos, carbonatado y sin carbonatar y sometido a cambios en la humedad de conservación.

c) En cuanto a la influencia del tipo de cemento se ha comprobado experimentalmente, mediante ensayos con disoluciones sintéticas, que simulan la fase acuosa del hormigón, que la velocidad de corrosión del galvanizado varía exponencialmente con el $\mathrm{pH}$, tal y como indicaban Roetheli, Cox y Littreal (fig. 8). Además se ha establecido, y este es el punto más importante por el momento, que existe un $\mathrm{pH}$ límite (o contenido en ion $\mathrm{Ca}^{2+}$ límite) por encima del cual (o por debajo, respectivamente) el hidroxicincato que se forma no es capaz de pasivar el galvanizado. Así, en la figura 9 se muestran las intensidades y los potenciales de corrosión de las disoluciones de la figura anterior a los 33 días de vida: por debajo de $\mathrm{pH}=13,1 \pm 0,2$ el galvanizado se ha pasivado completamente, y por encima de este valor la velocidad de corrosión se mantiene en valores tales que el recubrimiento galvanizado ha desaparecido en la mayoría de los casos.

En el caso de trabajar directamente con mortero, en la figura 10 se muestran las intensidades de corrosión de aceros galvanizados embebidos en morteros fabricados con 11 cementos diferentes (cuyos análisis químicos se dan en la Tabla 1). Puede comprobarse que en unos casos, al año del ensayo, el galvanizado está perfectamente pasivo, y en otros permanece en valores superiores a $0,1 \mu \mathrm{A} / \mathrm{cm}^{2}$ (equivalente a un ataque homogéneo de $1,5 \mu \mathrm{m} / a n ̃ o$, es decir, un recubrimiento de $60 \mu \mathrm{m}$ desaparecía en aproximadamente 40 años). 

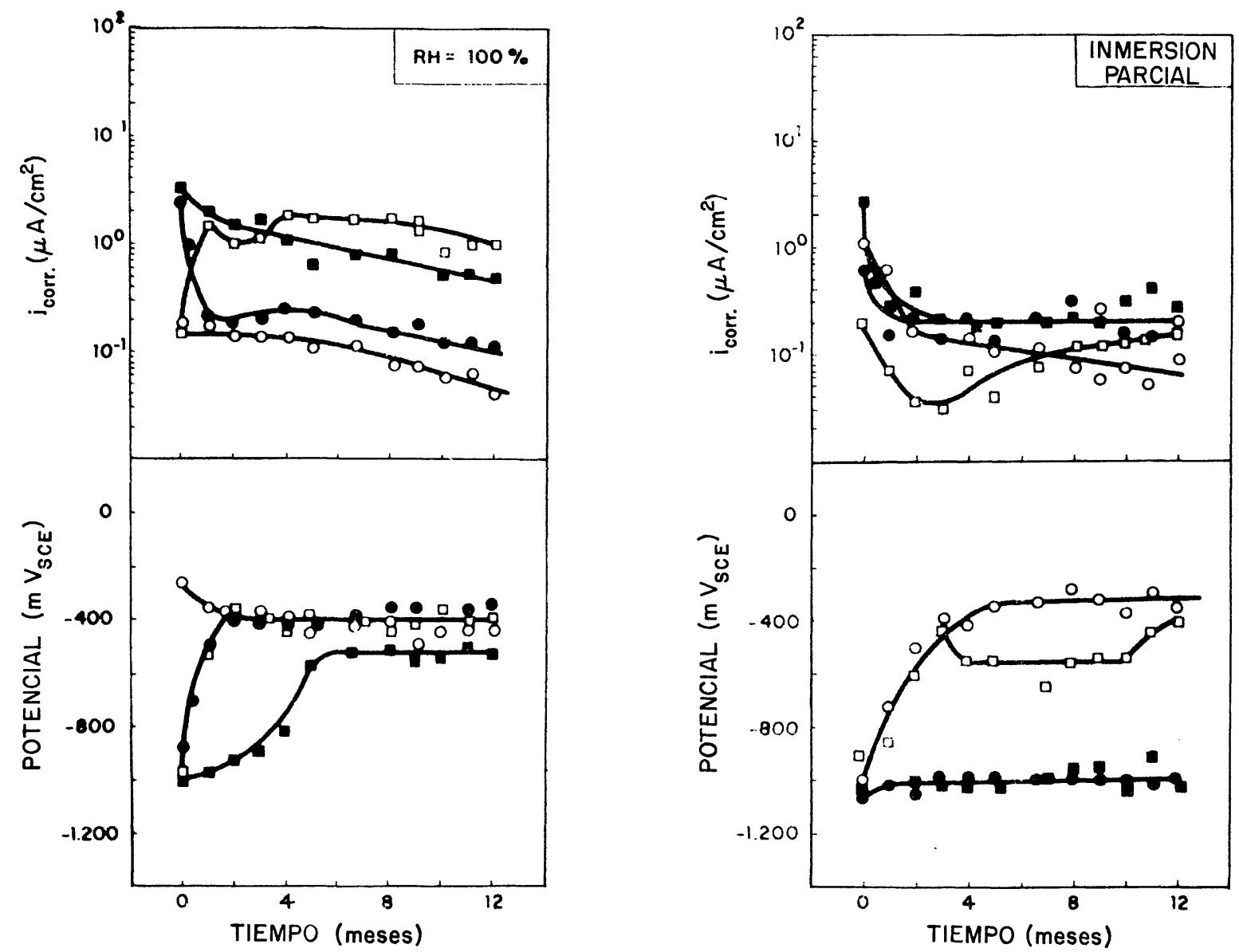

Fig. 7.- Variación de las intensidades y los potenciales de corrosión con el tiempo de aceros corrugados galvanizados embebidos en mortero carbonatado y sin carbonatar sometido en dos humedades de conservación.

o mortero sin aditivos.

- mortero carbonatado sin aditivos.

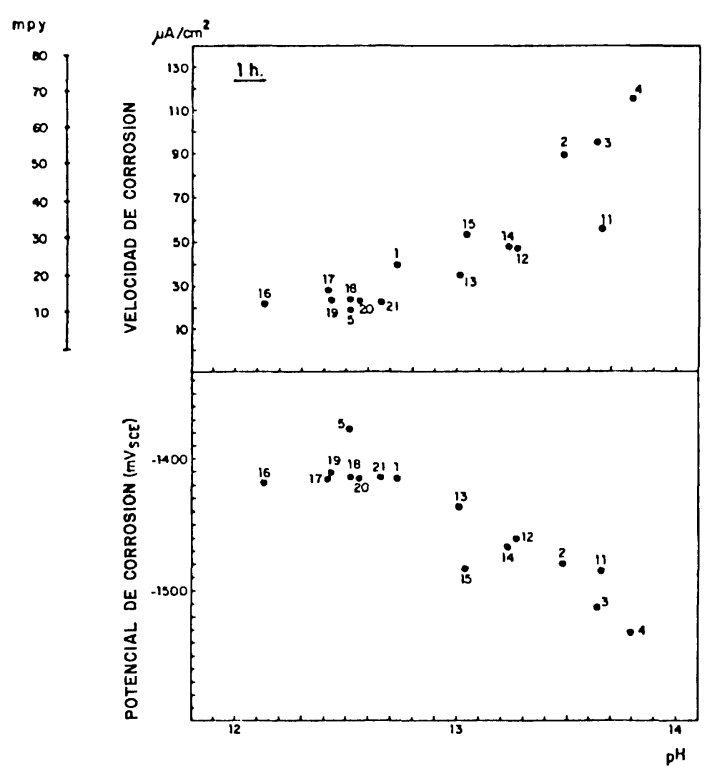

Fig. 8.- Valores de las intensidades y de los potenciales de corrosión a la $1 \mathrm{~h}$ de inmersión de aceros corrugados galvanizados en función del $\mathrm{pH}$ de las disoluciones de ensayo. $\square$ mortero con $2 \%$ de $\mathrm{Cl}_{2} \mathrm{Ca}$.

- mortero carbonatado con $2 \%$ de $\mathrm{Cl}_{2} \mathrm{Ca}$.

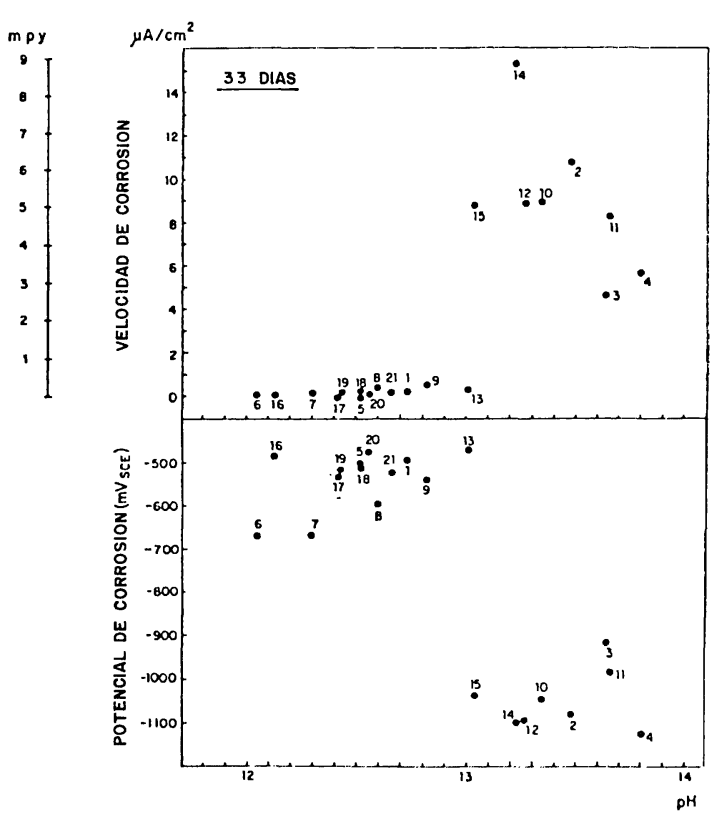

Fig. 9.-Idem. do la figura anterior a los 33 dias del ensayo. 
La dependencia del contenido en álcalis totales con el $\mathrm{pH}$ de una suspensión de cada tipo de cemento, se presenta en la figura 11, donde se comprueba que, para los cementos portland puros, el $\mathrm{pH}$ es casi una función lineal del contenido en álcalis. Los ce-

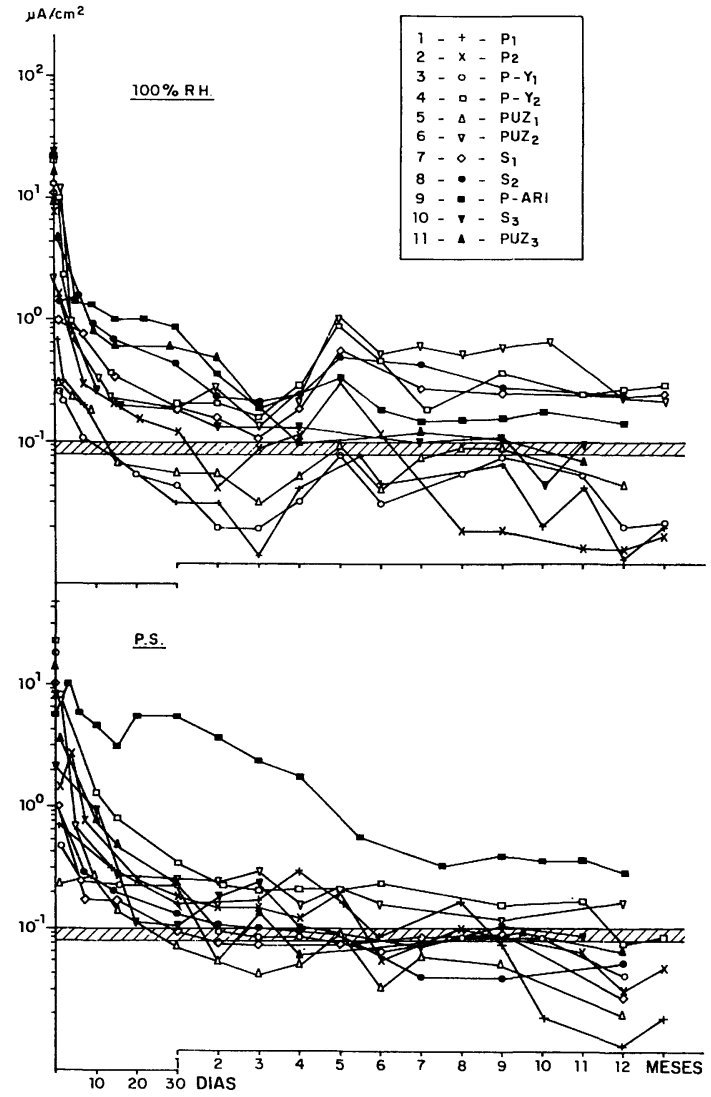

Fig. 10.-Variación de la intensidad de corrosión con el tiempo de aceros corrugados galvanizados embebidos en mortero fabricado con 11 cementos, cuyos análisis quimicos se dan en la tabla 1. mentos de adición suelen presentar $\mathrm{pH}$ menores, ya que no todos los álcalis que contienen se disuelven en agua.

En cuanto a la influencia de los $\mathrm{S}^{=}$contenidos en las escorias, es conocido que estos iones se oxidan en medios muy alcalinos, por lo que su efecto perjudicial debería desaparecer a las pocas horas del amasado. En teoría, estos iones son claramente agresivos para el galvanizado, por lo que en principio se les atribuye

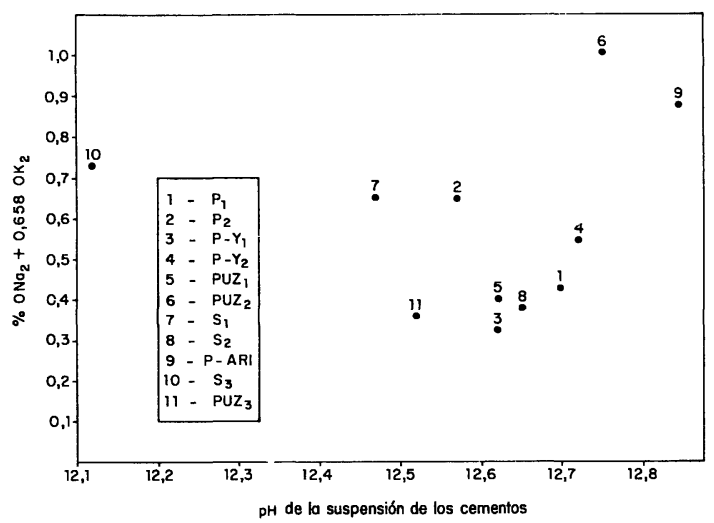

Fig. 11.-Valores del pH de suspensiones de cemento (relación $\mathrm{a} / \mathrm{c}=2$ ) en función del contenido de álcalis totales expresados como $\mathrm{Na}_{2} \mathrm{O}$ equivalente.

TA B L A 1

\begin{tabular}{|c|c|c|c|c|c|c|c|c|c|c|c|}
\hline Cementos & 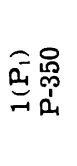 & 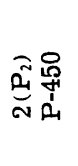 & 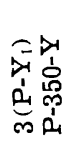 & 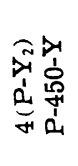 & 命品 & 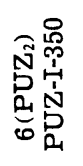 & 氛密 & 灾兽 & 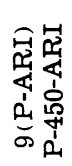 & 旋 & 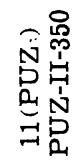 \\
\hline I.L. & 2,20 & 3,60 & 3,03 & 0,60 & 2,74 & 2,69 & - & - & 2,90 & - & 0,80 \\
\hline I.R. & 0,15 & 1,20 & 2,74 & 0,40 & 1,89 & 5,87 & 2,99 & 1,17 & 1,40 & 2,10 & 28,40 \\
\hline $\mathrm{SiO}_{2}$ & 20,00 & 18,50 & 20,40 & 20,10 & 21,28 & 25,77 & 19,95 & 19,97 & 18,60 & 30,60 & 17,60 \\
\hline $\mathrm{Al}_{2} \mathrm{O}_{3}$ & 6,02 & 4,90 & 4,53 & 4,30 & 5,05 & 6,91 & 4,67 & 7,54 & 4,80 & 9,20 & 6,10 \\
\hline $\mathrm{Fe}_{2} \mathrm{O}_{3}$ & 3,21 & 3,00 & 5,85 & 6,60 & 11,14 & 3,75 & 11,78 & 15,88 & 4,00 & 4,00 & 3,60 \\
\hline $\mathrm{OCa}$ & 64,00 & 63,30 & 58,93 & 64,30 & 52,39 & 46,96 & 52,24 & 48,14 & 62,30 & 43,90 & 40,20 \\
\hline OMg & 1,80 & 1,20 & 1,40 & 1,10 & 1,88 & 3,38 & 2,06 & 1,36 & 1,20 & 6,70 & 0,80 \\
\hline $\mathrm{SO}_{3}$ & 2,86 & 3,60 & 2,67 & 2,40 & 2,70 & 2,95 & 2,56 & 2,31 & 3,50 & 0,80 & 2,20 \\
\hline free $\mathrm{OCa}$ & 1,72 & 0,90 & 0,98 & 0,50 & 2,31 & 1,62 & 2,56 & 0,73 & 1,10 & 0.50 & 0,60 \\
\hline $\mathrm{Na}_{2} \mathrm{O}$ & 0,18 & 0,14 & 0,11 & 0,10 & 0,15 & 0,48 & 0,14 & 0,24 & 0,23 & 0,23 & 0,14 \\
\hline $\mathrm{K}_{2} \mathrm{O}$ & 0,39 & 0,78 & 0,30 & 0,68 & 0,37 & 0,84 & 0,36 & 0,63 & 1,00 & 0,76 & 0,34 \\
\hline MnO & & & & & & & & & & 0,029 & \\
\hline $\mathbf{S}=$ & & & & & & & & & & 0,70 & \\
\hline Suspensión & & & & & & & & & & & \\
\hline pH & 12,70 & 12,57 & 12,62 & 12,72 & 12,62 & 12,75 & 12,47 & 12,65 & 12,84 & 12,08 & 12.50 \\
\hline
\end{tabular}


las elevadas velocidades de corrosión que presentan los redondos en contacto con cementos con escorias que los contienen [figura $12(12)$ ] a pesar del bajo $\mathrm{pH}$ que se mide en las suspensiones de estos cementos.

Esta explicación sobre la influencia del contenido en álcalis de los cementos en la corrosión del galvanizado, ha permitido explicar los favorables resultados obtenidos en USA con este recubrimiento, donde los cementos, debido al riesgo de reactividad con determinados áridos suelen tener muy bajo contenido en álcalis, mientras que en Europa los resultados eran mucho más erráticos, al no estar en general controlado este factor.

En cuanto a la influencia de los cloruros en la corrosión del galvanizado solamente se quiere puntualizar que producen al igual que

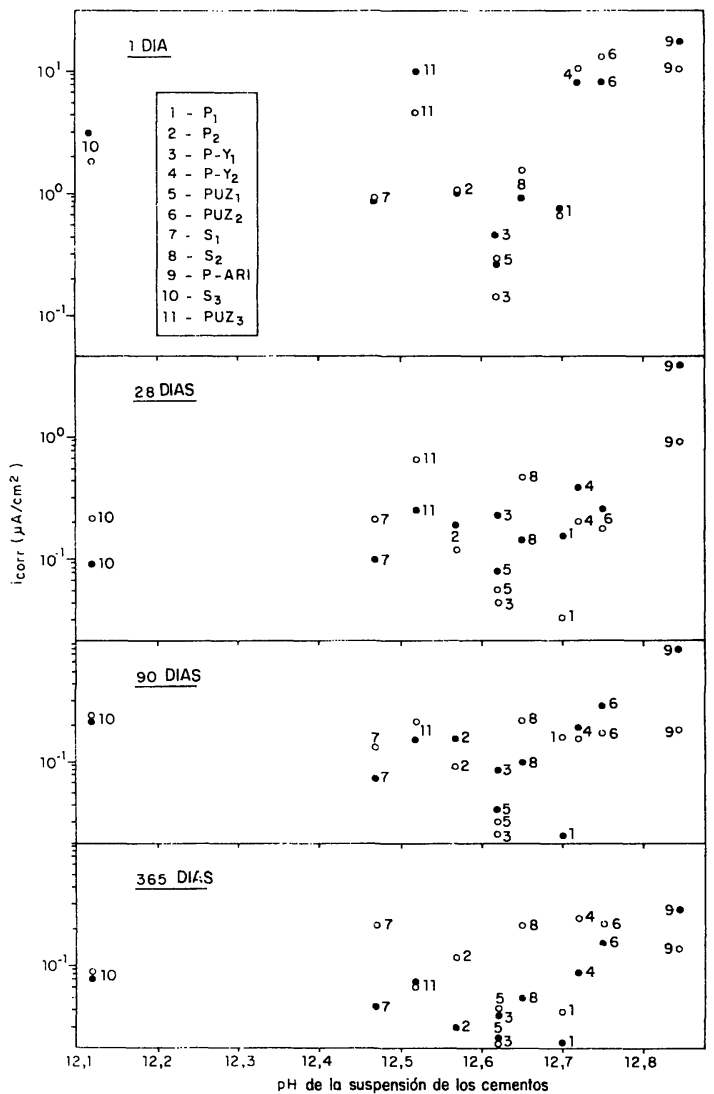

Fig. 12.-Valores de la intensidad de corrosión a $\operatorname{los} 1,28,90$ y 365 días de redondos corrugados galvanizados embebidos en mortero fabricado con 11 cementos diferentes. en el acero desnudo un ataque por picaduras, si bien el galvanizado es capaz de soportar sin corroerse una mayor proporción de estos iones.

De todos estos trabajos efectuados en el IETcc y el CENIM se pueden resumir como conclusiones, hasta el momento:

$1^{\circ}$ ) Para un mortero de relación $\mathrm{c} / \mathrm{a}=1 / 3$ y relación $\mathrm{a} / \mathrm{c}=0,5$ el límite de álcalis que asegura una corrosión suficientemente lenta del galvanizado es del 0,5\% expresado como $\mathrm{Na}_{2} \mathrm{O}$ equivalente. Este límite necesita posteriores precisiones, ya que el $\mathrm{pH}$ también depende de la proporción de cemento empleada la relación a/c y la humedad ambiental de conservación.

(Los autores J. Calleja y E. Molina no publican en su comunicación el análisis químico de los tres cementos utilizados en su ensayo, por lo que es imposible efectuar hipótesis sobre su comportamiento respecto de la corrosión del galvanizado. Además, al contener dos de ellos escorias siderúrgicas, habría que valorar también la influencia de los $\mathrm{S}^{=}$que pudiera contener).

2. ${ }^{\circ}$ El grosor del recubrimiento galvanizado tiene una importante incidencia sobre su corrosión, de tal forma que cuanto más gruesa y homógenea sea la capa $\eta$ de $\mathrm{Zn}$ puro, mayor es su resistencia al ataque.

3.") La corrosión sólo es importante cuando las humedades de conservación son superiores al 60-70\% ya que, por debajo de ellas, el hormigón se encuentra prácticamente seco, presentando una elevada resistividad óhmica por práctica ausencia de electrólito, que impide el desarrollo de las reacciones de corrosión.

4.") La carbonatación del hormigón no es perjudicial para el acero galvanizado (como lo es para el acero desnudo) ya que la intensidad del ataque (figuras 6 y 7) es del mismo orden o inferior que cuando está embebido en mortero sin carbonatar. 

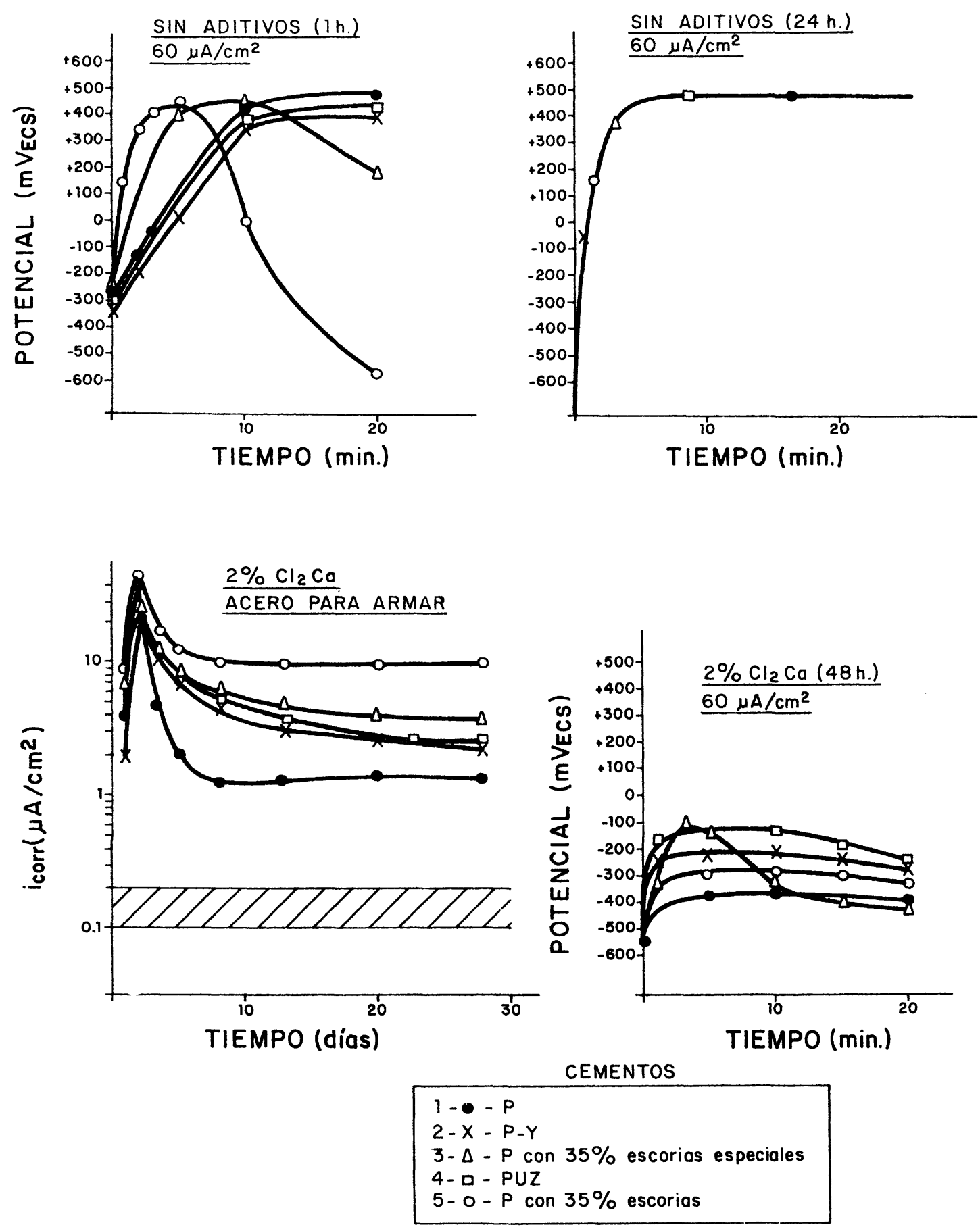

Fig. 13.-Curvas galvanostáticas y velocidad de corrosión de redondos de acero desnudo embebidos en mortero fabricado con 5 cementos diferentes.

La aplicación de estas conclusiones al caso de las tuberías galvanizadas, en contacto con hormigón o mortero, lleva a deducir que el que tantas de ellas se mantengan en buenas condiciones de conservación a lo largo de los años, es indudablemente debido, a que se han utilizado en general morteros pobres en cemento, que dan $\mathrm{pH}$ relativamente bajos inicialmente, y que además debido a la porosidad del material se han carbonatado con rapidez, lo que a la larga es beneficioso para la estabilidad del galvanizado.

Y cuando se han corroído, presumiblemente, o había cloruros en el mortero, o éste contenía materiales como el yeso, claramente agresivos para el $\mathrm{Zn}$, o la humedad ambiental se 
mantenía constantemente en valores elevados - lo que como se ha mostrado con anterioridad - supone un alto riesgo de corrosión para el galvanizado en contacto con hormigón, si le suman, el uso de un cemento con elevado contenido en álcalis solubles y un recubrimiento galvanizado de pequeño grosor o con defectos superficiales.

\section{INFLUENCIA DE LOS CEMENTOS CON ESCORIAS EN LA CORROSION DEL ACERO DESNUDO}

Al contrario que el galvanizado, el acero desnudo en contacto con hormigón se pasiva inmediatamente, es decir, se recubre de un óxido transparente, invisible y perfectamente compacto (al igual que el aluminio en contacto con la atmósfera) que le hace actuar casi como un metal noble. Para el acero desnudo lo más favorable es una elevada alcalinidad. El descenso de $\mathrm{pH}$ le resulta claramente perjudicial, ya que si la capa pasivante desaparece, el metal se corroe a muy elevada velocidad.

Si el mortero no contiene cloruros ni está carbonatado, el acero se mantiene pasivo indefinidamente y el tipo de cemento, o su diferente contenido en álcalis, no ejerce influencia detectable en su estabilidad.

En cuanto a la presencia de $\mathrm{S}^{=}$en las escorias, éstos dificultan en las primeras $24 \mathrm{~h}$ de amasado la formación de la capa pasivante y se detecta una velocidad de corrosión más elevada que con otros cementos de adición (5), figura 13. Si se determinan potenciales de ruptura, éstos se producen, es decir los $\mathrm{S}^{=}$son despasivantes para el acero.

Sin embargo, debido a la elevada alcalinidad, los $\mathrm{S}^{=}$se oxidan y al cabo de unas horas su efecto perjudicial desaparece y el cemento se comporta al igual que cualquier otro cemento de adición, es decir, es tanto más protector para el acero, cuanto mayor sea su contenido en clínker y viceversa (figura 13) (5).

Así pues, el mortero fabricado con cualquier tipo de cemento es un material protector perfectamente adecuado para las tuberías de acero desnudo. El problema surge cuando debido a una mala ejecución, el mortero u hormigón resulta pobre en cemento o poroso y se carbonata. Entonces el acero pierde su capa de pasividad y si la humedad ambiental es mayor del $60 \%$, se corroe a elevadas velocidades. En este caso, la velocidad de corrosión del acero es mayor en un mortero (carbonatado) fabricado con cemento con adiciones que en uno fabricado con portland puro (figura 14) (13).

La situación es similar si el ataque es por cloruros (figura 13), entonces el mismo acero, a igualdad del resto de las variables, se corroe a más velocidad si el mortero está fabricado con cementos con adiciones, que si lo está con portland puro (5).

De todas formas, en ambos casos, el cemento no será el responsable (el agente desencadenante) de la corrosión, sino que sería un mero agente acelerante. Es decir, en general, el "umbral" para que se produzca corrosión por carbonatación o por cloruros, es menor en el caso de los cementos con adiciones que en el de los portland puros, pero no se tiene noticia de casos fiables, donde el cemento sea el principal responsable de desencadenar la corrosión del acero desnudo (a excepción del cemento aluminoso).

Por otra parte, si el responsable fuera el cemento, en las zonas donde se han producido más casos de corrosión de tuberías, se deberían haber producido paralelamente accidentes por corrosión de armaduras.

64

Materiales DE ConstruCCION N. $187-1982$ 
Como resumen puede afirmarse que el mortero u hormigón que se mantiene alcalino y no contiene iones depasivantes (los $\mathbf{S}^{=}$ejercen sólo un efecto perjudicial pasajero) es un material protector idóneo para el acero desnudo, resultando inocua la humedad de conservación e indiferente el tipo de cemento.

Ahora, si el mortero contiene cloruros o se carbonata, entonces la velocidad de corrosión sí depende del tipo de cemento (que contenga o no adiciones) y de la humedad de conservación.

\section{ULTIMAS CONSIDERACIONES}

Un hecho que hemos comprobado sistemáticamente es que, cuando se fabrican probetas con armaduras en posición horizontal, por el fenómeno de exudación hacia la superficie de finos y líquido, se concentra éste en la parte inferior de los redondos, por lo que el ataque suele ser más intenso en esta zona. Cuando las armaduras se colocan verticales, el ataque no se localiza sistemáticamente en ninguna zona. Es de suponer que el fenómeno se produzca igual con las tuberías $\mathrm{y}$, por lo tanto, la parte inferior de los tramos horizontales sería donde el riesgo de corrosión es más elevado.

En cuanto al efecto corrosivo producido por el uso de papel de periódico para recubrir las tuberías que exponen en su trabajo los autores comentados (1), además de las causas por ellos apuntadas, de posible efecto de filtro y de reducción del $\mathrm{pH}$ (esta reducción del pH sería desfavorable para el acero negro, pero no para el galvanizado), hay que pensar también en un efecto de aireación diferencial que se produciría por una adherencia discontinua del papel al metal.

En el laboratorio, una de las técnicas a las que se acude para estudiar efectos de aireación diferencial, es precisamente recubrir con un determinado tipo de papel zonas del metal, que resultan así anódicas frente a las no recubiertas, que actúan de cátodos al tener un más fácil acceso del oxígeno hasta ellas.

Sólo un recubrimiento adherente, continuo y aislante de la humedad puede tener efectos beneficiosos, es decir, protectores. Todos los elementos que introduzcan discontinuidades, no sean adherentes y, por lo tanto, perfectos aislantes, suponen un aumento del riesgo de corrosión.

Como resumen, se quiere insistir en que, excepto en casos muy especiales, no es de esperar que sea el cemento el único o principal responsable de la corrosión de las tuberías. Esta suele ser la consecuencia de la concurrencia de varios factores desfavorables, en ge- 
neral, la presencia de cloruros y el uso de materiales inadecuados que resultan porosos y/o retienen humedad.

La corrosión de las tuberías puede ser evitada o minimizada si se extreman unas cuantas medidas de "buena práctica" que han sido recientemente resumidas por investigadores del CENIM, INCE e I.E.T.c.c. en un trabajo de próxima publicación (14) y en otro ya publicado (15).

\section{B I B I I O G R A F I A}

(1) J. Calleja, E. Molina: Corrosión de tuberias en instalaciones de edificación - Materiales de Construcción $n .^{\circ} 185$ (1982) 15

(2) M. Stern, E. Weisert: Experimental observations on the relation between Polarization Resistance and Corrosion Rate-Proc. Amer. Soc. Test. Mater. 59 (1959) 1280.

(3) C. Andrade, J. A. Gonzalez: Quantitative mesurements of corrosion rate of reinforcing steels embedded in concrete using polarization resistance measurements - Werkstoffe und Korrosion vol. 29 (1978) 515 .

(4) C. ANDRAde: Comentarios sobre factores desencadenantes y acelerantes de la corrosión de armaduras. Materiales de Construcción n. ${ }^{\circ} 172$ (1978) 71.

(5) C. Andrade, J. A. Gonzalez: Techniques éléctrochimiques qualitatives et quantitatives pour mésurer les effects des additions sur la corrosión des armatures - Congreso "Laitiers et ciments aux ajouts" Mons (Bélgica) sep. 1981 y traducido en Materiales de Construcción n. 182 (1981) 69.

(6) C. Andrade, A. Macias: Influence of alkali content of cement on corrosion of galvanized reinforcement - 13th Galvanizing Conference - Londres - Mayo (1982) y traducido en Materiales de Construcción n.* 184 (1981) 83.

(7) F. C. Porter: Reinforced concrete in Bermudas - Concrete 8 (1976) 29.

(8) M. Puech: Protection par le zinc des armatures pour bétons et fils de précontrainte - Annales de L'ITBTP n. 364 Sep. (1978) 82.

(9) J. A. Gonzalez, R. Vargas, C. Andrade: Revisión sobre el comportamiento de las armaduras galvanizadas en el hormigón - Hormigón y Acero n." li24 (1977) pág. 113.

(10) J. A. Gonzalez, C. Andrade: Effect of carbonation chlorides and relative ambient humidity on the corrosion of galvanized rebars embedded in concrete - British Corrosion Journal vol. 17 n. 1 (1982) 21.

(11) J. A. Gonzalez, A. J. Vazquez, C. Andrade: Les effects des cycles d'humidité sur la corrosion des armatures galvanisées dans les mortiers carbonatés et non carbonatés - Matériaux et Constructions vol. 15 n. 88 (1982) 271.

(12) C. Andrade, A. Molina, F. Huete, J. A. Gonzalez: Relation between the alkali content of cements and the corrosion rates of the galvanized reinforcements. Conference on Corrosion of Reinforcement in Concrete Construction - Londres, Junio (1983)

113) J. A. Gonzalez, C. Alonso, C. Andrade: Corrosion rate of reinforcements during accelerated carbonation of mortar made with different types of cement. Conference on Corrosion of Reinforcement in Concrete Construction - Londres, Junio (1983).

14) J. RodRiguez: Manual de Recomendaciones para prevenir la corrosión de tuberías de acero galvanizado en instalaciones de fontaneria. INCE.

(15) C. DEL Olmo: Durabilidad de las conducciones metálicas de agua en la edificación. Causas y remedios. Parte 1.' : Corrosión exterior de tuberias. Monografía n." 367 del IETcc. Diciembre 1981. 